\title{
Organic compounds in galaxies
}

\section{Takashi Onaka $^{1}$, Hiroko Matsumoto ${ }^{1}$, Itsuki Sakon ${ }^{1}$, and Hidehiro Kaneda ${ }^{2}$}

\author{
${ }^{1}$ Department of Astronomy, Graduate School of Science, The University of Tokyo, Bunkyo-ku, \\ Tokyo 113-0033, Japan \\ email: onaka@astron.s.u-tokyo.ac.jp, matsumoto@astron.s.u-tokyo.ac.jp, \\ isakon@astron.s.u-tokyo.ac.jp \\ ${ }^{2}$ Institute of Space and Astronautical Science, Japan Aerospace Exploration Agency, \\ Sagamihara, Kanagawa 229-8510, Japan \\ email: kaneda@ir.isas.jaxa.jp
}

\begin{abstract}
The unidentified infrared (UIR) emission bands in the near- to mid-infrared are thought to originate from organic compounds in the interstellar medium. Recent space observations with Spitzer and AKARI have clearly revealed that the UIR bands are commonly seen in external galaxies, including elliptical galaxies, except for very metal-poor dwarf galaxies. They are also detected in extended structures of galaxies, such as extra-planar components and filaments produced by outflows, suggesting that the band carriers are ubiquitous organic compounds in galaxies. Since the UIR bands are prominent features in the infrared spectrum of galaxies and are linked to the star-formation activity, it is highly important to understand the nature, formation, processing, and destruction of the UIR band carriers in galaxies. While there is no systematic variation detected in the UIR spectrum in normal galaxies, significantly low values are derived for the ratio of the $7.7 \mu \mathrm{m}$ to $11.2 \mu \mathrm{m}$ bands in elliptical galaxies as well as in galaxies with low-luminosity AGNs compared to normal star-forming galaxies. Relatively low band ratios are also seen in the UIR band spectrum of extended structures in galaxies. If the same mechanism leads to the low band ratio, it would provide important information on the band carrier properties. It should also be noted that the band carriers are believed to be destroyed in a short time scale in environments where low band ratios are detected. The survival and supply processes in these environments are a key to understand the nature of the band carriers.
\end{abstract}

Keywords. Galaxies: ISM, infrared: galaxies, infrared: ISM, dust, extinction

\section{Introduction}

A set of emission bands at $3.3,6.2,7.6-7.8,8.6,11.2,12.7$, and $16-18 \mu \mathrm{m}$, have been observed in various celestial objects and are called the unidentified infrared (UIR) bands. The exact nature of the carriers has not yet been understood completely, but it is generally believed that the emitters or emitting atomic groups containing polycyclic aromatic hydrocarbons (PAHs) or PAH-like atomic groups of carbonaceous materials are responsible for the UIR bands. The UIR bands have been observed in a wide range of objects and they are commonly seen in diffuse Galactic emission (Onaka 2004) as well as in external galaxies (Helou et al. 2000, Smith et al. 2007), suggesting that the band carriers are a major component of the interstellar matter. Since their intensities are well correlated to far-infrared emission or to star-formation activity (Onaka 2000, Peeters et al. 2004) and since the UIR bands are distinct features in the infrared spectrum of galaxies, the understanding of their origin and nature is of great importance for the study of star-formation activities in remote galaxies as well. 
Where the band carriers are formed and how they are processed and destroyed in the interstellar medium (ISM) are important issues for the study of the UIR bands in addition to the exact nature of the carriers. The band carriers may be formed in circumstellar envelopes of carbon stars (Frenklach \& Feigelson 1989, Latter 1991, Cherchneff et al. 1992, Galliano et al. 2008a). The UIR bands have, however, been detected only in a handful of carbon stars (Boersma et al. 2006, Speck et al. 2008). It is not yet clearly understood how efficiently the carriers are formed in stellar sources. They could also be produced by fragmentation of large carbonaceous grains in the interstellar medium (Jones et al. 1996, Greenberg et al. 2000) or formed in situ in dense clouds (Herbst 1991).

Small band carriers are thought to be destroyed rapidly in hot plasma by thermal sputtering (Dwek \& Arendt 1992, Tielens et al. 1994). They will also be destroyed in strong radiation fields (Allain et al. 1996), although the radiation destruction is only effective in the vicinity of exciting sources. Peeters et al. (2002) indicate that there are at least three classes of the 6-9 $\mu \mathrm{m}$ UIR band spectrum in Galactic sources on the basis of the peak wavelengths of the bands. Processing from 'fresh' band carriers to 'matured' ones is not detected in a planetary nebula, suggesting that the change must occur in the ISM (Matsumoto et al. 2008a). On the other hand, the relative band strengths and profiles of the UIR bands do not show systematic variations in the mid-infrared (MIR) spectrum of diffuse Galactic radiation (Chan et al. 2001, Kahanpää et al. 2003) except that small changes are seen between those in the inner and outer Galaxy (Sakon et al. 2004). External galaxies provide a much wider range of physical conditions and thus variations with environments should appear more clearly, if any. In this study, the variations of the UIR band spectrum among external galaxies that include elliptical and dwarf galaxies together with their spatial variations in galaxies are discussed based on latest observations with Spitzer (Werner et al. 2004) and AKARI (Murakami et al. 2007).

\section{UIR Bands in Galaxies}

Elliptical galaxies are deficient in interstellar matter and they are believed to provide hostile environments against dust grains: they are associated with hot plasma, which destroys dust grains in a short time scale by thermal sputtering. Recent Spitzer and $A K A R I$ observations, however, indicate that the presence of the UIR bands is not uncommon in elliptical galaxies although the appearance of the UIR bands is quite different from those familiar to spiral galaxies (Kaneda et al. 2005, 2007). Figure 1 shows some examples of spectra taken with the Infrared Spectrograph (IRS) on board Spitzer (Houck et al. 2004) and the Infrared Camera (IRC) on board AKARI (Onaka et al. 2007, Ohyama et al. 2007). The $11.2 \mu \mathrm{m}$ band is clearly seen together with the $17 \mu \mathrm{m}$ complex, whereas the $6.2,7.7$, and $8.6 \mu \mathrm{m}$ bands are faint or almost absent (see also Kaneda et al. 2008a,b).
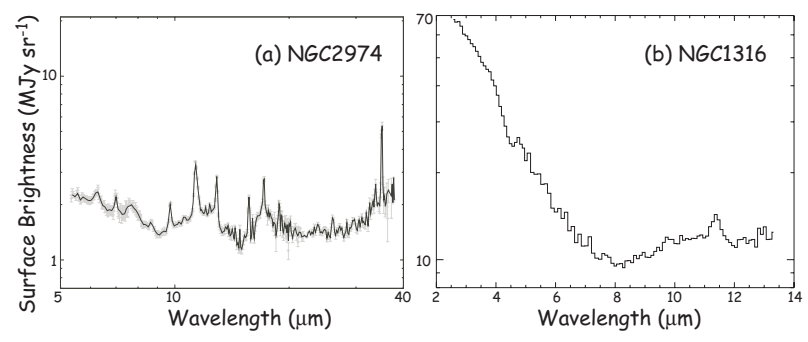

Figure 1. Examples of elliptical galaxy spectra. (a) NGC2974 with IRS on board Spitzer (Kaneda et al. 2005) and (b) NGC1316 with IRC on board AKARI (Kaneda et al. 2007). 


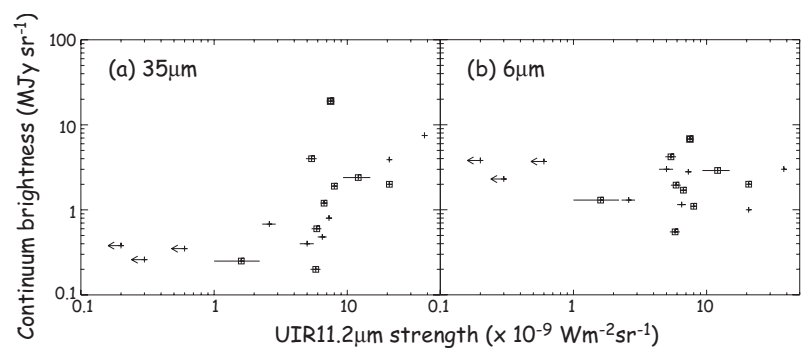

Figure 2. Correlation of the continuum emission with the UIR $11.2 \mu \mathrm{m}$ strength for elliptical galaxies (Kaneda et al. 2008b) (a) $35 \mu \mathrm{m}$ continuum emission vs. the UIR band and (b) $6 \mu \mathrm{m}$ continuum emission vs. the UIR band. The boxes indicate galaxies with low-luminosity AGNs.

The $3.3 \mu \mathrm{m}$ band is shown to be absent in NGC1316 based on AKARI IRC observations (Figure 1b), suggesting the dominance of large neutral PAHs (Kaneda et al. 2007).

While the presence of the UIR bands in elliptical galaxies is well established, the formation and survival of the band carriers in them are not yet clearly understood. There seem to be three potential origins for the band carriers in elliptical galaxies; stellar mass loss, recent merger events, and cooling flows. Kaneda et al. (2008b) indicate that the band intensity is well correlated with the dust continuum emission at $35 \mu \mathrm{m}$, but the correlation is poor with the continuum emission at $6 \mu \mathrm{m}$ for 18 elliptical galaxies (Figure 2). Since the $6 \mu \mathrm{m}$ continuum is supposed to come from stellar photospheric emission in these objects, it is suggested that the stellar mass loss is not a dominant source for the UIR band carriers in elliptical galaxies. Correlation with the dust continuum strongly suggests the interstellar origin of the band carriers.

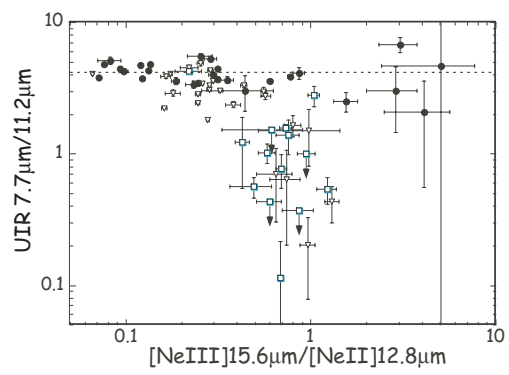

Figure 3. Band intensity ratio of the UIR 7.7 to $11.2 \mu \mathrm{m}$ against the line ratio of [NeIII]15.6 $\mu \mathrm{m} /[\mathrm{NeII}] 12.8 \mu \mathrm{m}$. The SINGS galaxy data are taken from Smith et al. (2007). They are indicated either by the filled circles for those with HiI-dominated nuclei or by the open triangles for those with low-luminosity AGNs. Elliptical galaxies from Kaneda et al. (2008) are indicated by the open squares. The dotted line shows the median of the HiI-dominated sources.

Smith et al. (2007) studied the UIR band spectra of the Spitzer Infrared Nearby Galaxies Survey (SINGS) sample galaxies (Kennicutt et al. 2003). Figure 3 shows the band ratio for the SINGS galaxies together with the elliptical galaxies (open squares) in Kaneda et al. (2008b). Smith et al. (2007) found the intensity ratio of the 7.7 to $11.2 \mu \mathrm{m}$ UIR bands is lower for galaxies harboring low-luminosity AGNs (open triangles) than galaxies with HII-dominated nuclei (filled circles). Since most elliptical galaxies harbor AGNs, this trend is compatible with the results of elliptical galaxies as indicated in Figure 3. The important question is whether or not AGNs play a key role in making the band ratio low. Kaneda et al. (2007) point out that the AGN activity of NGC1316, which shows a low band ratio in its MIR spectrum (Figure 1b), ceased 100 Myr ago and thus its AGN should not make an appreciable effect on the present UIR band spectrum of this galaxy. 
Recently Shi et al. (2007) show that the UIR band ratios of AGN host galaxies are not different from those of star-forming galaxies. Taking account of these facts, it seems to be unlikely that AGNs play a major role for the low band ratio. There must be other factors that produce the peculiar UIR band spectrum.

Figure 3 also shows that the band ratio, if the UIR bands are present, does not change even for dwarf galaxies with hard radiation fields characterized by high $[\mathrm{NeIII}] /[\mathrm{NeII}]$ ratios. On the other hand, observations clearly indicate that the UIR bands are absent in metal-poor dwarf galaxies. There seems to be a threshold, such as $\log (\mathrm{O} / \mathrm{H})+12 \sim 8$, in the metallicity, below which the UIR bands are not present (Smith et al. 2007, Engelbracht et al. 2008). Since those low-metallicity galaxies also have very strong radiation fields, the absence of the UIR bands could be related either to the metallicity, the radiation field strength, or to a combination of them (Wu et al. 2006). On the other hand, O'Halloran et al. (2006) attributed the absence to efficient destruction of the band carriers in shocks based on the correlation with the [FeII]/[NeII] line ratio. Recently Galliano et al. (2008a) indicated that if carbonaceous dust is mainly supplied from carbon stars, young galaxies may not have an appreciable amount of carbonaceous dust since they do not have a sufficient time for less massive stars to evolve to carbon stars. The absence of the UIR bands can thus be accounted for by the delayed formation of carbonaceous dust in low-metallicity environments. Spitzer and $A K A R I$ observations have revealed that the UIR band carriers are commonly present in most galaxies including ellipticals except for metal-poor dwarf galaxies and that they are major organic compounds in the ISM.

\section{Spatial Variation of the UIR bands in Galaxies}

Sakon et al. (2004) reported the first detection of a systematic variation in the UIR bands in diffuse Galactic emission that the UIR 7.7 to $11.2 \mu \mathrm{m}$ band ratio is slightly lower in the outer Galactic plane than in the inner region based on observations of diffuse Galactic emission with IRTS (Onaka et al. 1996). Sakon et al. (2007) also indicated that the band ratio is lower in the interarm region than in the arm region in NGC 6946 based on observations with $A K A R I$ IRC (see Figure 4). Galliano et al. (2008b) made detailed analysis on ISOCAM observations of several galaxies, suggesting that the UIR 7.7 to $11.2 \mu \mathrm{m}$ band ratio is lower in the outer region than in the disk region of galaxies. Irwin \& Madden (2006) reported the detection of the UIR bands in the halo of NGC 5907 and suggested that the 7.7 to $11.2 \mu \mathrm{m}$ band ratio becomes lower in the halo region than in the disk region. ISO and Spitzer observations further suggest that the UIR bands are present in regions even several kpc away from the galactic disk (Engelbracht et al. 2006, Irwin et al. 2007). The environmental conditions at such distant regions are not favorable for the band carriers and the survival of the carriers is an interesting problem to be investigated.

Recently Matsumoto et al. (2008b) reported the detection of the UIR bands in the MIR spectrum of one of the $\mathrm{H} \alpha$ filaments in the dwarf galaxy NGC 1569 (Hunter et al. 1993)

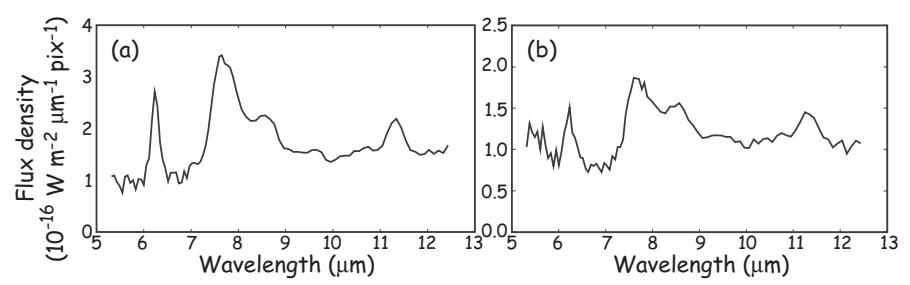

Figure 4. AKARI IRC spectra of (a) arm and (b) interarm region of NGC 6946 (Sakon et al. 2007). 
based on $A K A R I$ IRC observations. NGC 1569 is known to harbor several super star clusters (e.g. Tokura et al. 2006) and the latest star-formation event is estimated to have occurred about 10 Myr ago (Greggio et al. 1998). The UIR band emission is detected over the entire galactic disk (Madden et al. 2006). HI gas inflow from an outer cloud is also suggested, which may have triggered the star-formation and the creation of the $\mathrm{H} \alpha$ filaments (Stil \& Israel 1998, Mühle et al. 2005). The X-ray distribution shows a good correspondence to the $\mathrm{H} \alpha$ filaments, suggesting that the filaments are produced by the outflow from the galaxy disk triggered by the recent star-formation activity (Martin 1998, Martin et al. 2002).

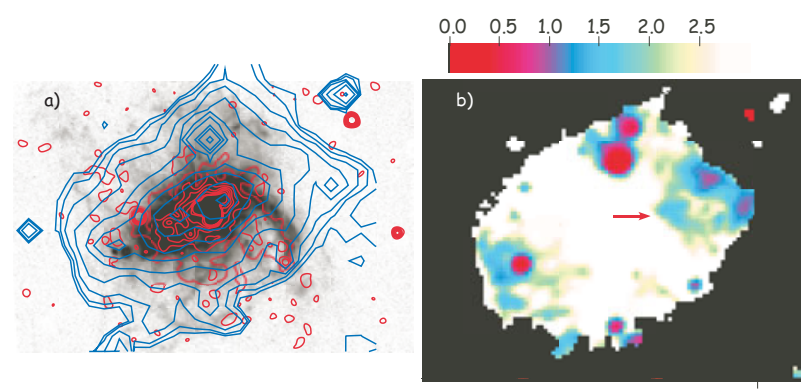

Figure 5. (a) Images of NGC 1569. The blue contours indicate the $7 \mu \mathrm{m}$ band image of the AKARI IRC observations and the red contours show the X-ray intensity taken by Chandra (Martin et al. 2002). The gray scale shows the $\mathrm{H} \alpha$ image taken from Hunter et al. (1993). (b) The 15 to $7 \mu \mathrm{m}$ band ratio image of NGC 1569. The arrow indicates the position of the filamentary structure corresponding to the $\mathrm{H} \alpha$ filament.

Figure 5a shows the $7 \mu \mathrm{m}$ band intensity of the $A K A R I$ IRC in blue contours together with the $\mathrm{H} \alpha$ in gray scale and the X-ray emission in red contours (see the color figure in the electronic version). A prominent $\mathrm{H} \alpha$ filament seen in the west part of the galaxy encloses the X-ray emission, just outside of which a filamentary structure is seen in the $7 \mu \mathrm{m}$ contours. Figure 5b shows an image of the 15 to $7 \mu \mathrm{m}$ color. The arrow indicates the position of the filamentary structure corresponding to the $\mathrm{H} \alpha$ filament. The filament appears bright at $7 \mu \mathrm{m}$ relative to $15 \mu \mathrm{m}$. The IRC $7 \mu \mathrm{m}$ band effectively probes the UIR 6.2 and $7.7 \mu \mathrm{m}$ bands and the $15 \mu \mathrm{m}$ band intensity comes mostly from the MIR continuum emission (Sakon et al. 2007). Figure 5b suggests the presence of the UIR bands in the filament.

Subsequent spectroscopic observations with the IRC confirmed the presence of the UIR bands in the MIR spectrum (Matsumoto et al. 2008b). Figure 6 shows the comparison of the filament spectrum with that in the galaxy disk taken by Spitzer IRS. After fitting with Lorentzians (e.g. Smith et al. 2007), the intensity ratio of the 7.7 and $11.2 \mu \mathrm{m}$ bands is derived to be $3.5 \pm 0.4$ and $1.4 \pm 0.6$ for the disk and the filament, respectively. The band ratio of the galaxy disk is compatible with those of star-forming galaxies (Figure 3), whereas that of the filament shows as lower a value as seen in extended structures of other galaxies. The UIR $7.7 \mu \mathrm{m}$ band consists of at least two components, $7.6 \mu \mathrm{m}$ and $7.8 \mu \mathrm{m}$. A closer look of the spectrum further indicates that the $7.8 \mu \mathrm{m}$ component seems to be absent in the filament spectrum. A similar "narrow" and weak $7.7 \mu \mathrm{m}$ band has been reported for the low-metallicity dwarf galaxy IC2574 (Walter et al. 2007). Further investigations are needed to understand the link between the two objects.

The age of the filamentary structure is estimated to be several Myr based on the expansion velocity of the $\mathrm{H} \alpha$ filament $\left(\sim 90 \mathrm{~km} \mathrm{~s}^{-1}\right.$, Westmoquette et al. 2008$)$ and the distance to the filament from the youngest super star cluster $(\sim 320 \mathrm{pc})$, which is consistent 

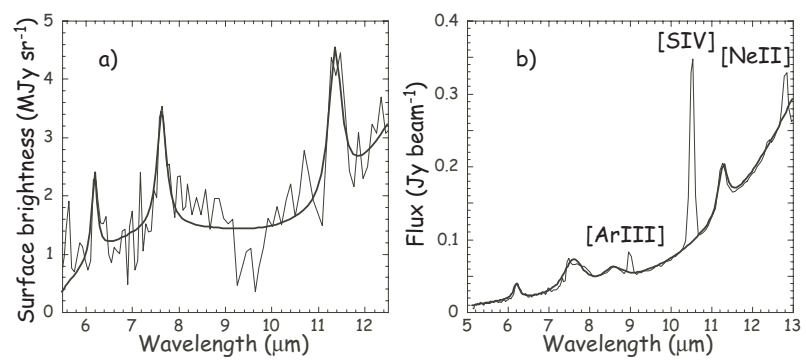

Figure 6. (a) AKARI IRC spectrum of a $\mathrm{H} \alpha$ filament of NGC 1569 (Matsumoto et al. 2008b) and (b) Spitzer IRS spectrum of the NGC 1569 disk (Tajiri et al. 2008). The thin lines indicate the observed spectra, while the thick lines indicate the fitted spectra. The ionic forbidden lines are labeled.

with the age of the recent star-formation event. The destruction time scale by thermal sputtering in hot plasma can be approximated by $\sim 1 \times 10^{3} \mathrm{yr}(a / 1 \mathrm{~nm})\left(1 \mathrm{~cm}^{-3} / n_{\mathrm{H}}\right)$ for $10^{6}<T<3 \times 10^{8} \mathrm{~K}$, where $a$ is the particle radius and $n_{\mathrm{H}}$ is the proton density (Tielens et al. 1994). The density of the X-ray emitting gas is estimated to be $0.035 \mathrm{~cm}^{-3}$ (Ott et al. $2005 \mathrm{a}, \mathrm{b})$. Thus for $a=1 \mathrm{~nm}$, the destruction time scale becomes about $3 \times 10^{4} \mathrm{yr}$, too short compared to the age of the filament. There must be an efficient supply source for the band carriers in the filament region. No significant star-formation is seen in the region and thus neither formation in dense clouds nor production in the stellar outflow seems to be a dominant supply source. It is most likely that the carriers are produced by fragmentation from large carbonaceous grains in shocks, which produce the $\mathrm{H} \alpha$ emission.

\section{Summary}

Recent Spitzer and AKARI observations provide new and interesting data for the study of the UIR bands in external galaxies. They have clearly revealed that the UIR bands are commonly seen in galaxies, including elliptical galaxies, except for very metalpoor dwarf galaxies. They also detect the UIR bands in extra-planar structures and filamentary structures that are formed by outflows from the galactic disk. The UIR bands have been detected even several kpc away from the galactic disk, indicating the UIR band carriers are major and ubiquitous organic compounds in the universe. The formation, processing, and destruction of the band carriers in various environments are key issues for the understanding of the UIR bands.

The ratio of the UIR 7.7 to $11.2 \mu \mathrm{m}$ band intensity is quite constant for star-forming galaxies, including dwarf galaxies with strong radiation fields. However the ratio is systematically lower in extended structures of galaxies. It is also indicated to be low in the interarm region. The extreme case is seen in elliptical galaxies, where some of their spectra do not show the 6.2 and $7.7 \mu \mathrm{m}$ emission at all. The low value is usually attributed to the low ionization degree of the band carriers in these environments since the 6.2 and $7.7 \mu \mathrm{m}$ bands are not strong in neutral PAHs and are enhanced when they become ionized. The objects that show low band ratios have a common characteristic that they are mostly low density and free from significant stellar components. Some of them are associated with $\mathrm{H} \alpha$ emission and/or X-ray emission. If these low ratios have a common origin, it would be very important to understand the physical conditions that make the ratio low for the study of the UIR band carriers. There is also an indication that the $7.7 \mu \mathrm{m}$ band lacks its longer wavelength component in some objects when they are weak. High $\mathrm{S} / \mathrm{N}$ ratio spectra are required for further study. 
The band carriers are easily destroyed in hot plasma and thus the presence of the UIR bands in extended structures of galaxies suggests revisit of the survival/destruction process of the band carriers. Recent observations also indicate likely supply sources for the UIR band carriers in galaxies. Correlation study of the elliptical galaxy sample indicates that mass loss from evolved stars is not a dominant source. It is suggested that fragmentation from large carbonaceous grains play a role in a filament of NGC1569.

\section{Acknowledgements}

This work is based on observations with Spitzer, which is operated by Jet Propulsion Laboratory, California Institute of Technology, under NASA contract 1407, and on observations with $A K A R I$, a JAXA project with the participation of ESA. The authors thank all the members of the $A K A R I$ project and the members of the Interstellar Medium and Nearby Galaxy team for their continuous encouragement. This work was supported by a Grant-in-Aid for Scientific Research on Priority Areas from the Ministry of Education, Culture, Sports, Science and Technology, Japan and Technology of Japan and a Grant-in-Aid for Scientific Research from the Japan Society of Promotion of Science.

\section{References}

Allain, T., Leach, S., \& Sedlmayr, E. 1996, A\&A, 305, 602

Boersma, C., Hony, S., \& Tielens, A. G. G. M. 2006, A\&A, 447, 213

Chan, K.-W. et al. 2001, ApJ, 546, 273

Cherchneff, I., Barker, J. R., \& Tielens, A. G. G. M. 1992, ApJ, 401, 269

Dwek, E. \& Arendt, R. G. 1992, ARAA, 30, 11

Engelbracht, C. W. et al. 2006, ApJ (Letters), 642, L127

Engelbracht, C. W. et al. 2008, ApJ, 678, 804

Frenklach, M. \& Feigelson, E. D. 1989, ApJ, 341, 372

Galliano, F., Dwek, E., \& Chanial, P. 2008a, ApJ, 672, 214

Galliano, F., Madden, S. C., Tielens, A. G. G. M., Peeters, E., \& Jones, A. P. 2008b, ApJ, 679,310

Greenberg, J. M. et al. 2000, ApJ (Letters), 531, L71

Greggio, L. et al. 1998, ApJ, 504, 725

Helou, G. et al. 2000, ApJ (Letters), 532, L21

Herbst, E. 1991, ApJ, 366, 133

Hunter, D. A., Hawley, W. N., \& Gallagher, J. S. 1993, AJ, 106, 1797

Houck, J. R. et al. 2004, ApJS, 154, 18

Irwin, J. A., Kennedy, H., Parkin, T., \& Madden, S. 2007, A\&A, 474, 461

Irwin, J. A. \& Madden, S. 2006, A\&SA, 445, 123

Jones, A. P., Tielens, A. G. G. M., \& Hollenbach, D. J. 1996, ApJ, 469, 740

Kahanpää, J., Mattila, K., Lehtinen, K., Leinert, C., \& Lemke, D. 2003, A\&̋A, 405, 999

Kaneda, H., Onaka, T., \& Sakon, I. 2005, ApJ (Letters), 632, L83

Kaneda, H., Onaka, T., \& Sakon, I. 2007, ApJ (Letters), 666, L21

Kaneda, H., Onaka, T., Sakon, I., Matsumoto, H., \& Suzuki, I. 2008a, this volume

Kaneda, H., Onaka, T., Sakon, I., Kitayama, T., Okada, Y., \& Suzuki, T. 2008b, ApJ, in press

Kennicutt, R. C. Jr. et al. 2003, PASP, 115, 928

Madden, S., Galliano, F., Jones, A. P., \& Sauvage, M. 2006, A\&\&A 446, 877

Martin, C. L. 1998, ApJ, 491, 561

Martin, C. L., Kobulnicky, H. A., \& Heckman, T. M. 2002, ApJ, 574, 663

Matsumoto, H. et al. 2008a, ApJ, 677, 1120

Matsumoto, H., Onaka, T., Sakon, I., \& Kaneda, H. 2008b, this volume

Mühle, S., Klein, U., Wilcots, E. M., \& Hüttenmeister, S. 2005, AJ, 130, 524

Murakami, H. et al. 2007, PASJ, 59, S369

O'Halloran, B., Satyapal, S., \& Dudik, R. P. 2006, ApJ, 641, 795 
Ohyama, Y. et al. 2007, PASJ, 59, S411

Onaka, T. 2000, Adv. Sp. Res., 25, 2167

Onaka, T. 2004, in: A. N. Witt, G. C. Clayton, \& B. T. Draine (eds.), Astrophysics of Dust, ASP Conf. ser. 309, p. 163

Onaka, T., Yamamura, I., Tanabé, T., Roellig, T. L., \& Yuen, L. 1996, PASJ (Letters), 48, L59

Onaka, T. et al. 2007, PASJ, 59, S401

Ott, J., Walter, F., \& Brinks, E. 2005a, MNRAS, 358, 1423

Ott, J., Walter, F., \& Brinks, E. 2005b, MNRAS, 358, 1453

Peeters, E., Spoon, H. W. W., \& Tielens, A. G. G. M. 2004, ApJ, 613, 986

Peeters, E. et al. 2002, A\&A, 390, 1089

Sakon, I. et al. 2004, ApJ, 609, 203 (Erratum: ApJ, 625, 1062)

Sakon, I. et al. 2007, PASJ, 59, S483

Shi, Y. et al. 2007, ApJ, 669, 841

Smith, J. D. et al. 2007, ApJ, 656, 770

Speck, A., Barlow, M., Wesson, R., Glayton, G., \& Volk, K. 2008, this volume

Stil, J. M. \& Israel, F. P. 1998, A\&AA, 337, 64

Tajiri, Y. Y. et al. 2008, in: R. Chary, H. I. Teplitz, \& K. Sheth (eds.), Infrared Diagnostics of Galaxy Evolution, ASP Conf. ser. 381, p. 50

Tielens, A. G. G. M., McKee, C. F., Seab, C. G., \& Hollenbach, D. J. 1994, ApJ, 431, 321

Tokura, D. et al. 2006, ApJ, 648, 355

Walter, F. et al. 2007, ApJ, 661, 102

Werner, M. W. et al. 2004, ApJS, 154, 309

Westmoquette, M. S., Smith, L. J., \& Gallagher, J. S. 2008, MNRAS, 383, 864

Wu, Y. et al. 2006, ApJ, 639, 157

\section{Discussion}

PAPOULAR: To my knowledge, the effect of UV or visible radiation, if any, is to aromatize the particles. So what is the experimental laboratory evidence for a possible destruction of these particles to explain the disappearance of some of the features?

ONAKA: I do not know very well about the laboratory data, but observationally there are data indicating the UIR bands are not present in the $\mathrm{H}$ II regions. But there are some exceptions also, as there are data indicating the presence of the UIR bands together with the $[\mathrm{Ne} \mathrm{II}]$ line emission. But there are also data indicating that they are quite absent inside diffuse $\mathrm{H}$ II regions.

PAPOULAR: Yes, this is an observational effect, but that does not mean that the radiation can destroy these particles.

Ceccarelli: I am curious you didn't show the sizes. How far away from the galactic plane you see these PAHs?

ONAKA: They are somewhere around several kilo parsecs from the galactic plane of M82. 